\title{
Effect of obesity intervention programs on adipokines, insulin resistance, lipid profile, and low-grade inflammation in 3- to 5-y-old children
}

\author{
Gianni Bocca', Eva Corpeleijn², Ronald P. Stolk ${ }^{2}$, Bruce H. Wolffenbuttel ${ }^{3}$ and Pieter J. Sauer ${ }^{1}$
}

BACKGROUND: Childhood obesity can cause the development of cardiovascular risk factors. We assessed the effect of a multidisciplinary intervention program on cardiovascular risk factors and compared this effect with a usual-care program in 3- to 5-y-old overweight or obese children.

METHODS: Seventy-five children were randomly assigned to a multidisciplinary intervention or a usual-care program. Anthropometry, body composition, and abdominal adipose tissue were assessed at the start and end of a 16-wk program. Concurrently, fasting concentrations of serum lipids, glucose, insulin, HbA1c, leptin, adiponectin, high-sensitive C-reactive protein (hsCRP), tumor necrosis factor (TNF)- $\alpha$, and interleukin (IL)-6 were determined.

RESULTS: In both groups, insulin sensitivity improved, demonstrated by decreased insulin concentrations and a decreased HOMA2-IR. In the multidisciplinary intervention group, there was also a decrease of HbA1c and TNF- $\alpha$. In the usual-care group, an increase in glucose concentrations was found. Comparing both groups, changes over time were not different, besides trends in the decrease in total cholesterol and TNF- $\alpha$, in favor of the multidisciplinary intervention group. Combining the results of both groups, a correlation was found between the decrease in body fat percentage (BF\%), and both HOMA2-IR and triglyceride (TG) concentrations.

CONCLUSION: In 3- to 5-y-old children, both obesity intervention programs improved insulin sensitivity, in parallel with a reduced $\mathrm{BF} \%$.

$\mathbf{R}^{\mathrm{e}}$ ecent data have shown that the prevalence of childhood overweight and obesity in the Netherlands is still increasing (1). Childhood obesity is a risk factor for the development of insulin resistance (IR), dyslipidemia, and hypertension at a later age, together described as metabolic syndrome (MS) (2). MS cannot be diagnosed in children under the age of $10 \mathrm{y}$ (3). However, increased concentrations of the separate components of MS have already been demonstrated in children aged 6-9 y (4).

Systemic low-grade inflammation may be an underlying cause of MS, induced by the release of inflammatory cytokines from adipose tissue, and even more so from visceral adipose tissue $(5,6)$. Increased concentrations of tumor necrosis factor (TNF)- $\alpha$, high-sensitive C-reactive protein (hsCRP), and interleukin (IL)-6 have been found in obese children aged 9-13 y $(7,8)$. The adipose tissue hormone adiponectin has anti-inflammatory properties, demonstrated by the significant inverse correlation with markers of inflammation in obese children (8). Whether these deleterious processes related to obesity are already present in overweight and obesity at a very young age is unknown, and no data on systemic low-grade inflammation are available in very young obese children.

Multidisciplinary lifestyle intervention programs for obese children have proven to be successful in reducing weight (9). Recent studies demonstrated that weight loss in obese children aged 6-11 y also reduced low-grade inflammation and IR $(4,8)$. In obese children, 9-13 y old, weight loss over a 1-y period led to a significant decrease in triglyceride (TG) concentrations and an improvement of an indicator of insulin sensitivity, the homeostasis model assessment of IR (HOMA-IR) (7). This study did not show a significant correlation between changes in inflammatory parameters and changes in lipids and HOMA-IR. Significant weight loss can lead to increased concentrations of adiponectin, as has been demonstrated in obese children aged 6-15 y (10).

Little is known about the effects of a successful treatment program for obesity on markers of low-grade inflammation, lipid profile, IR, and adipokines in preschool-aged children. We assessed the effects of a 16-wk multidisciplinary intervention program, aimed at reducing BMI, on inflammatory parameters, lipid profile, IR, and adipokines, in 3- to 5-y-old overweight or obese children and compared the results with a group of overweight or obese children receiving a usual-care program.

\section{RESULTS}

During the treatment period, 13 children were lost to followup: 7 children in the multidisciplinary intervention group and 
6 children in the usual-care group. Children who were either lost to follow-up or stopped the study had a slightly lower BMI compared with children who continued the study. However, this difference was not statistically significant. At the end of the treatment period, children in the multidisciplinary intervention group showed a larger decrease in BMI $(-1.2(1.0)$ $\mathrm{kg} / \mathrm{m}^{2}$, mean (SD) vs. $\left.-0.6(1.1)\right)$, BMI $z$-score $(z$-BMI) $(-0.5$ $(0.4)$ vs. $-0.3(0.4))$ and waist circumference $z$-score $(z$-WC) $(-0.3(0.5)$ vs. $0.0(0.6))$ compared with children in the usualcare group, as previously described (11). Also, children in the multidisciplinary intervention group showed a statistically significant decrease in body fat percentage (BF\%) $(-1.5(3.4))$, in contrast to no statistically significant decrease observed in the usual-care group $(-0.3(4.0))$. However, the mean difference in the change in $\mathrm{BF} \%$ between both groups was not statistically significant (11). Table 1 shows the details at baseline on serum lipids, parameters for glucose metabolism, and markers for low-grade systemic inflammation and adipokines, for the multidisciplinary intervention group and usual-care group. At baseline, there were no significant differences for these parameters between the two groups.

Table 2 shows the changes in parameters for glucose metabolism, markers for low-grade systemic inflammation, and adipokines and serum lipids for both groups, between baseline and the end of the 16-wk treatment program, and the difference in the change in these parameters between the groups. In the multidisciplinary intervention group, a statistically significant decrease in insulin, updated HOMA-IR (HOMA2-IR), HbA1c and TNF- $\alpha$ was found. IL- 6 showed a trend toward a statistically significant decrease $(P=0.09)$. In the usual-care group, decreases were only found for insulin and HOMA2-IR, in parallel with a small but statistically significant increase in serum glucose $(4.4 \mathrm{mg} / \mathrm{dl})$. Comparing both groups, changes over time were not significantly different apart for trends in the decrease in total cholesterol (T (C) $)(P=0.07)$ and TNF- $\alpha(P=$ 0.06 ), which almost reached statistical significance in favor of the multidisciplinary intervention group.

We investigated in an observational manner whether the changes over time in measures of adiposity were related to improvements in cardiovascular risk factors. These analyses were performed in children from both treatment groups together, since children lost weight in both programs. Moreover, there were no differences between these variables at baseline. Regarding parameters for glucose metabolism, statistically significant correlations were found between the decrease in $\mathrm{BF} \%$ and decrease in insulin $(r=0.352 ; P=0.02)$ and between the decrease in $\mathrm{BF} \%$ and improvement in HOMA2-IR $(r=0.365 ; P$ $=0.02)$ (Figure 1a). There were no statistically significant correlations between changes in visceral fat (VF) and changes in insulin concentrations $(r=0.011 ; P=0.94)$ and HOMA2-IR $(r=0.026 ; P=0.87)$. Concerning the lipid profile, a decrease in BF\% correlated with a decrease in serum TG $(r=0.290$; $P=0.02$ ) (Figure $1 b$ ), mainly due to a correlation between changes in abdominal subcutaneous fat and TG $(r=0.273$; $P=0.04)$. No statistically significant correlations were present between a decrease in VF and a decrease in T (C) $(r=0.187$;
$P=0.15)$ and TG $(r=0.085 ; P=0.52)$. Statistically significant correlations were found between changes in leptin concentrations and changes in $z$-BMI $(r=0.535 ; P<0.001), z$-WC $(r=0.323$; $P=0.01$ ), VF, and BF\% (both $r=0.345 ; P=0.01$ ). No correlation was found between changes in $\mathrm{BF} \%$ and changes in adiponectin concentrations $(r=-0.181 ; P=0.16)$.

Regarding associations between parameters for glucose metabolism and markers for inflammation and adipokines, statistically significant correlations were found between changes in leptin concentrations and changes in insulin concentrations $(r=0.367 ; P=0.01)$ and HOMA2-IR $(r=0.372 ; P=0.01)$. Also, a decrease in hsCRP concentrations showed a correlation with a decrease in HOMA2-IR $(r=0.359 ; P=0.03)$ and with a decrease in insulin concentrations $(r=0.360 ; P=0.02)$. For associations between serum lipids and markers for lowgrade systemic inflammation, an inverse correlation was found between changes in IL- 6 and changes in serum high-density lipoprotein (cholesterol) (HDL (C)) $(r=-0.311 ; P=0.02)$ and between the change in IL- 6 and change in T (C) $(r=-0.346$; $P=0.01$ ).

In the factors analysis, we included changes in $z$-BMI, leptin, adiponectin, HOMA2-IR, HDL (C), T (C) and TG, TNF- $\alpha$, IL-6, and hsCRP. Low-density lipoprotein (cholesterol) was excluded because it inhibited the elimination process. The factor analysis was performed several times with including and excluding several factors, to check for stability of the analysis. The final model included all variables but low-density lipoprotein (cholesterol) and resulted in four factors with eigenvalue $>1$ that together explained $53 \%$ of the variance, i.e., a leptin/ BMI factor (17\%), a $\mathrm{T}(\mathrm{C})$ factor (13\%) that also included HDL (C) and TNF- $\alpha$, a TG factor (13\%) with HDL (C) and HOMA2-IR, and a IL-6/hsCRP factor (10\%) that also included HOMA2-IR.

\section{DISCUSSION}

This study shows that both obesity treatment programs in 3- to 5 -y-old children resulted in improved insulin sensitivity. In the children receiving the multidisciplinary intervention program, TNF- $\alpha$, an indicator of low-grade systemic inflammation, decreased. When combining both groups, strong correlations were found between improvements in parameters for glucose metabolism and lipid profile, with improvements in body composition. Also, correlations were found between a decrease in inflammatory markers and improvements in lipid profile and glucose metabolism.

The importance of reducing IR in lowering metabolic risk factors in children has been demonstrated in a previous study (12). A decrease in HOMA-IR correlated with a decrease in TG, independently of changes in weight status, thereby emphasizing the role of IR in the development of cardiovascular risk factors. The association between reduced body fat and decreased IR was further illustrated by a prospective cohort study in obese children, aged 9-17 y (13). In obese Hispanic adolescents participating in an exercise program, a decrease in VF also decreased IR (14). Our data confirm these findings and show that this association is already present in very young children. 


\section{Articles $\mid$ Bocca etal.}

Table 1. Descriptive and anthropometric characteristics, serum lipids, adipokines, and markers for low-grade inflammation and insulin resistance of the study population at baseline

\begin{tabular}{|c|c|c|c|}
\hline Characteristics & $\begin{array}{l}\text { Multidisciplinary intervention } \\
\text { group }(n=40)\end{array}$ & $\begin{array}{l}\text { Usual-care group } \\
\qquad(n=35)\end{array}$ & $P$ value \\
\hline Age (years) & $4.6(0.8)$ & $4.7(0.8)$ & 0.47 \\
\hline \multicolumn{4}{|l|}{ Adiposity (ref. 11) } \\
\hline BMI z-score & $2.7(1.0)$ & $2.7(1.0)$ & 0.77 \\
\hline Fat-free mass (kg) & $19.7(2.4)$ & $19.7(3.6)$ & 0.97 \\
\hline Visceral fat (cm) & $4.4(1.4)$ & $4.3(0.8)$ & 0.65 \\
\hline Subcutaneous fat (cm) & $1.8(0.7)$ & $1.7(0.7)$ & 0.64 \\
\hline \multicolumn{4}{|l|}{ Lipids } \\
\hline Triglycerides (mg/dl) & $63.6(23.4)$ & $75.3(41.3)$ & 0.13 \\
\hline \multicolumn{4}{|l|}{ Glucose metabolism } \\
\hline Glucose (mg/dl) & $75.6(5.1)$ & $74.9(7.5)$ & 0.67 \\
\hline Insulin (mU/l) & $8.1(4.3)$ & $7.6(3.8)$ & 0.67 \\
\hline HOMA2-IR & $1.0(0.5)$ & $1.0(0.5)$ & 0.68 \\
\hline $\mathrm{HbA1c}(\%)$ & $5.3(3.3-6.0)$ & $5.3(4.5-5.8)$ & 0.78 \\
\hline \multicolumn{4}{|l|}{ Inflammation/adipokines } \\
\hline Adiponectin (ng/ml) & $18.9(5.0)$ & $17.6(4.3)$ & 0.26 \\
\hline
\end{tabular}

Normal distributed variables are expressed as mean ( \pm SD); non-normally distributed variables are expressed as median (minimum to maximum). For differences between groups, the independent Student's $t$-test was used for variables with a normal distribution and the Mann-Whitney test for non-normally distributed variables.

Obesity-related dyslipidemia, already described in childhood, increases the risk on the development of atherosclerosis (15). Lifestyle intervention programs are recommended to treat child and adolescent overweight and obesity (16). To date, little is known about the effect of lifestyle intervention programs on lipid profiles in preschool-aged children. Recently, it was shown that a 13-wk multicomponent wellness intervention program in, on average 16 y olds, led to a $15 \%$ increase in HDL (C) concentrations, despite a lack of decrease in BMI (17). In 9- to 13-y-old obese children, weight loss over a 1-y period was associated with a significant decrease of TG concentrations (7). Our study in 3- to 5-y-old overweight and obese children shows that a reduction in abdominal subcutaneous fat and $\mathrm{BF} \%$ is associated with a decrease in TG concentrations. These results may imply that treatment programs in preschool-aged overweight and obese children can prevent a cumulative lifetime exposure to increased risk factors for cardiovascular disease.

The present study also shows that a multidisciplinary intervention program in preschool-aged overweight and obese children has positive effects on one of the markers of lowgrade systemic inflammation, TNF- $\alpha$. Positive effects of a reduction in BMI on TNF- $\alpha$ have been described in obese children with a mean age of $10.7 \mathrm{y}$ (8). In contrast, no effect on TNF- $\alpha$ concentrations was observed in 9- to 13-y-old obese children who experienced a decrease in BMI SD score of $\geq 0.5$ over a 1-y period (7). For comparison, in the present study, the decrease in the multidisciplinary treatment group was $0.5 \mathrm{SD}$ over $16 \mathrm{wk}$ and $0.3 \mathrm{SD}$ in the usual-care group. A significant decrease in CRP and IL- 6 concentrations was demonstrated in 24 obese children, aged 6-9 y old, who obtained a decrease in BMI SD score of $\geq 0.5$ over a period of 9 mo (4). This could not be confirmed by our data, although the decrease in IL- 6 concentrations in the multidisciplinary intervention group showed a trend toward statistical significance.

Obesity can have effects on cardiovascular risk profile and IR through several mechanisms. The two most well-known pathways are adipocyte dysfunction/inflammation and lipotoxicity/ectopic fat disposition (18). Inflammatory factors have been shown to induce IR in several tissues by interacting with the 
Table 2. Changes in lipids, adipokines, and markers for low-grade inflammation and insulin resistance, between baseline and end of the 16-wk treatment program

\begin{tabular}{|c|c|c|c|c|c|c|}
\hline \multirow[b]{2}{*}{ Characteristics } & \multicolumn{2}{|c|}{$\begin{array}{c}\text { Multidisciplinary intervention } \\
\text { group }\end{array}$} & \multicolumn{2}{|c|}{ Usual-care group } & \multicolumn{2}{|c|}{$\begin{array}{l}\text { Difference between } \\
\text { groups }\end{array}$} \\
\hline & $\begin{array}{c}\text { Mean } \\
\text { difference (SD) }\end{array}$ & $P$ value & $\begin{array}{c}\text { Mean } \\
\text { difference (SD) }\end{array}$ & $P$ value & Mean (SD) & $P$ value \\
\hline Total cholesterol (mg/dl) & $-2.5(16.2)$ & 0.38 & $6.4(20.3)$ & 0.11 & $8.9(4.7)$ & 0.07 \\
\hline High-density lipoprotein (cholesterol) (mg/dl) & $-0.2(9.6)$ & 0.89 & $0.7(9.5)$ & 0.70 & $0.9(2.5)$ & 0.71 \\
\hline Low-density lipoprotein (cholesterol) (mg/dl) & $-3.6(15.3)$ & 0.19 & $3.0(17.1)$ & 0.36 & $6.7(4.2)$ & 0.12 \\
\hline Glucose (mg/dl) & $1.9(7.1)$ & 0.21 & $4.4(8.9)$ & 0.03 & $2.5(2.3)$ & 0.29 \\
\hline Insulin (mU/l) & $-2.2(4.0)$ & 0.01 & $-2.9(4.8)$ & 0.01 & $0.6(1.3)$ & 0.63 \\
\hline HOMA2-IR & $-0.3(0.5)$ & 0.02 & $-0.4(0.6)$ & 0.01 & $0.1(0.2)$ & 0.65 \\
\hline $\mathrm{HbA1c}(\%)$ & $-0.3(0.5)$ & 0.01 & $-0.1(0.5)$ & 0.42 & $0.2(0.1)$ & 0.12 \\
\hline Tumor necrosis factor- $\alpha$ (pg/ml) & $-2.5(4.9)$ & 0.01 & $-0.4(3.1)$ & 0.48 & $2.1(1.1)$ & 0.06 \\
\hline Interleukin-6 (pg/ml) & $-0.4(1.3)$ & 0.09 & $0.0(1.0)$ & 0.83 & $0.4(0.3)$ & 0.23 \\
\hline
\end{tabular}

Positive numbers indicate an increase over time. For within-group differences, a paired sample Student's t-test was used for normally distributed variables. For variables not normally distributed, a related-samples Wilcoxon signed-rank test was used.

insulin signaling pathway $(19,20)$. An obesity-related profile of inflammation and adipocyte dysfunction is often found in parallel with impairments in lipid metabolism (21). Elevated FFA are thought to play an important role, since higher circulating fatty acids may induce intramyocellular lipid accumulation, directly inducing IR in skeletal muscle and impairing substrate utilization (22). All of these processes are well described in adults. It is surprising to see that even at a very young age, these processes are ongoing.

Since it can be hypothesized that the pathophysiological pathways could still be different at this young age, we explored the data using factor analysis. The finding that $43 \%$ of the variance was explained by a factor dominated by changes in leptin/BMI, T (C), and TG suggests that changes in lipotoxicity concomitant with a reduction in leptin are the most important changes with weight loss in 3- to 5-y-old children. The remaining factor was dominated by changes in IL-6 and hsCRP (10\%) suggesting that also inflammation plays a role, but to a lesser extent, and that it is not strongly intertwined with the lipotoxicity pathway. Of course, these factors are exploratory in nature and should only be interpreted as hypothesis generating.

Strong points of this study are the young age of the children and the range in degree of obesity of the study participants. Until present time, no data were available on the effect of obesity intervention programs on cardiovascular risk factors in preschool-aged children. Our data may imply that children with overweight or obesity at this young age will benefit from obesity intervention programs with respect to reducing the risk of developing MS in adulthood. Furthermore, since children in our study were either overweight or obese, the results on adipokines, IR, lipid profile, and low-grade inflammation can be generalized to a larger extent, in contrast to studies only reporting on children with extremes in obesity.

A weak point of the study was the limited power to detect more differences between baseline and the end of the treatment program. Due to low power for changes in leptin, adiponectin, HDL (C), IL-6, and hsCRP, the lack of significant effects should be interpreted with care. Nevertheless, we feel that our data clearly show beneficial effects of both obesity treatment programs in preschool-aged children. Also, the present study did not include a control group of nonoverweight children to determine if the results of the parameters are different from a normal population or to evaluate if there is a change over time in children without overweight. It was our intention to evaluate the effect of an intervention program in overweight or obese children. Therefore, we did not include children without overweight.

In addition, the use of bioimpedance analysis to estimate $\mathrm{BF} \%$ and the use of ultrasound to measure abdominal fat have some limitations, although discriminatory power for repeated measurements is good. Moreover, it has been demonstrated in 6-y-old children that bioimpedance analysis is reliable in assessing fat mass (23). Furthermore, the use of ultrasound to measure abdominal adiposity has been validated in adults (24) and proven its reliability in assessing subcutaneous abdominal fat in 6- to 7-y-old children (25).

In conclusion, the present study shows that obesity treatment programs in 3- to 5-y-old children have beneficial effects on insulin sensitivity and that, already in very young children, a reduction in body fat improves insulin sensitivity. In the multidisciplinary intervention group, next to improved insulin sensitivity, we also found a reduction in TNF- $\alpha$ concentrations. It can be hypothesized that the improvement in these 
parameters may diminish the increased cumulative lifetime exposure to risk factors related to childhood obesity, thereby reducing MS and cardiovascular disease risk in adulthood. Prospective studies are needed to further explore the effects of different treatment programs for overweight and obesity in preschool children, on parameters leading to MS later in life.

\section{METHODS}

Children and parents participated in a randomized controlled clinical trial, called "Groningen Expert Center for Kids with ObesityOutpatient Clinic." Details on this study have been described previously (11). In brief, children aged 3-5y were referred to the outpatient clinic by youth health care physicians, general practitioners, or other physicians if they had a $z$-BMI $>1$.1. Children with medical conditions causing obesity, eating disorders, mental retardation, or behavioral problems were excluded from the study. Enrollment took place from October 2006 to March 2008, and a total of 75 children were included.

Children and their parents were randomly assigned to a multidisciplinary intervention or a usual-care program. The outline of both programs is given in Table 3. The multidisciplinary intervention program consisted of dietary advice, physical activity sessions, and psychological counseling for parents. Dietary advice, six sessions of $30 \mathrm{~min}$ each, was given by a dietician and focused on improving eating behavior by setting personal goals for parents and children. Feedback was given on these goals using food diaries. Physical activity, 12 sessions of $60 \mathrm{~min}$ each, were supervised by a physiotherapist and resembled elementary school exercise, including ball playing and dancing to music. An active lifestyle was propagated during these activity sessions. Psychological counseling for parents, six sessions of $120 \mathrm{~min}$ each, was given by a psychologist and aimed at teaching parents to be a healthy role model, thereby demonstrating a healthy lifestyle to their children. In the usual-care program, children and parents were seen by a resident in pediatrics (three sessions of 30-60 min each) who advised on healthy eating and physical activity. In total, both programs lasted $16 \mathrm{wk}$. Written informed consent was obtained from the parents or legal caretakers. The study was approved by the Medical Ethics Committee of the University Medical Center Groningen.

At the start and at the end of the treatment period, anthropometry and assessment of body composition were performed. Height and weight were measured using standard calibrated stadiometers and scales and used to calculate BMI. WC was measured using a standard measuring tape, at the midpoint between the lateral iliac crest and the lowest rib. The web-based program Growth Analyser 3 (Growth Analyser B.V., Rotterdam, The Netherlands) was used to calculate age- and gender-specific $z$-BMI and $z$-WC. Body composition, i.e., fat-free mass and $\mathrm{BF} \%$, was assessed by bioimpedance analysis (BIA101; Akern S.r.l./RJL Systems, Florence, Italy), and measurements of VF and subcutaneous fat were performed using ultrasound (SonoSite Titan; SonoSite, Bothell, WA) (24).
Blood was drawn after an overnight fast, at the start and end of the treatment period. An enzymatic colorimetric method (Roche Modular, Mannheim, Germany) was used to determine $\mathrm{T}(\mathrm{C})$, HDL (C), low-density lipoprotein (cholesterol), and TG. HbAlc was determined using a high-performance liquid chromatography method (Bio-Rad Variant; Bio-Rad Laboratories, Veenendaal, The
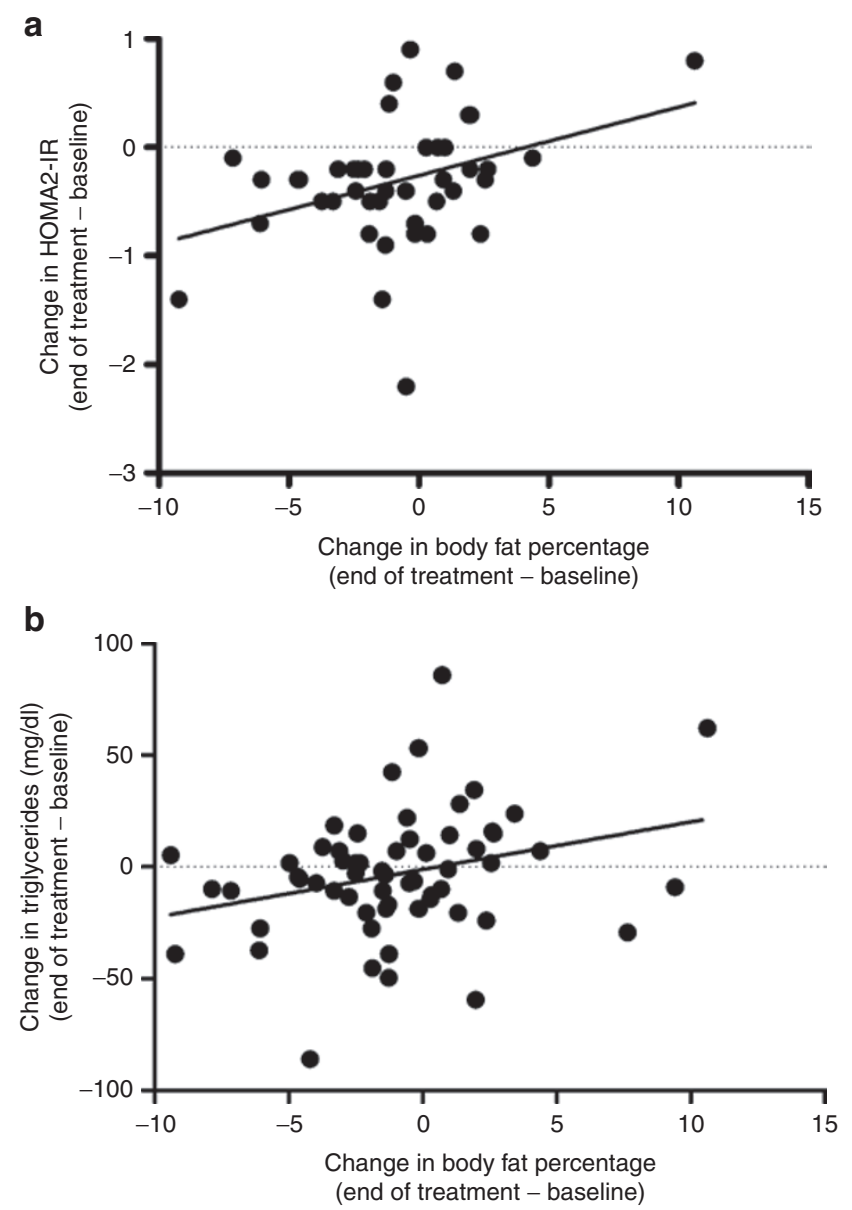

Figure 1. (a) Pearson correlation coefficient between the change in body fat percentage and the change in the updated HOMA-IR, between baseline and end of the treatment period. $P=0.02 ; r^{2}=0.133$. (b) Pearson correlation coefficient between the change in body fat percentage and the change in serum triglycerides $(\mathrm{mg} / \mathrm{dl})$, between baseline and end of the treatment period. $P=0.02 ; r^{2}=0.084$.

Table 3. Schematic representation of weekly activities in the multidisciplinary intervention and usual-care groups

\begin{tabular}{|c|c|c|c|c|c|c|c|c|c|c|c|c|c|c|c|c|c|}
\hline $\begin{array}{l}\text { Multidisciplinary } \\
\text { intervention group }\end{array}$ & Baseline & 1 & 2 & 3 & 4 & 5 & 6 & 7 & 8 & 9 & 10 & 11 & 12 & 13 & 14 & 15 & 16 \\
\hline Measurements & $x$ & - & - & - & - & - & - & - & - & - & - & - & - & - & - & - & $x$ \\
\hline Dietary advice by dietician & - & $X$ & $x$ & - & $x$ & - & - & - & $x$ & - & - & $x$ & - & - & $x$ & - & - \\
\hline $\begin{array}{l}\text { Physical activity session by } \\
\text { physiotherapist }\end{array}$ & - & - & - & $x$ & $x$ & $x$ & $\mathrm{X}$ & $\mathrm{x}$ & $\mathrm{x}$ & $x$ & $\mathrm{x}$ & $x$ & $x$ & $x$ & $x$ & - & 一 \\
\hline $\begin{array}{l}\text { Parental behavioral } \\
\text { therapy by psychologist }\end{array}$ & - & - & - & - & - & $x$ & - & $\mathrm{x}$ & - & $\mathrm{x}$ & - & $x$ & - & $x$ & - & $x$ & - \\
\hline Usual-care group & Baseline & 1 & 2 & 3 & 4 & 5 & 6 & 7 & 8 & 9 & 10 & 11 & 12 & 13 & 14 & 15 & 16 \\
\hline Measurements & $x$ & - & - & - & - & - & - & - & - & - & - & - & - & - & - & - & $x$ \\
\hline $\begin{array}{l}\text { Visit to resident in } \\
\text { pediatrics }\end{array}$ & - & - & $\mathrm{X}$ & - & - & - & - & $\mathrm{x}$ & - & - & - & - & $x$ & - & - & - & - \\
\hline
\end{tabular}

Numbers indicate the weeks of the program. X indicates visit. 
Netherlands), insulin by radioimmunoassay (Diagnostic Systems Laboratories, Webster, TX), and glucose by an enzymatic method (hexokinase-mediated reaction; Roche Modular, Mannheim, Germany). HOMA2-IR was used to calculate IR (26). For markers of low-grade systemic inflammation, hsCRP was determined by a nephelometric method (BN II system; Siemens Healthcare Diagnostics B.V., Breda, The Netherlands). Serum concentrations of leptin, IL-6, and TNF- $\alpha$ were simultaneously measured by a combination enzyme-linked immunosorbent assay (Milliplex Map Human Adipokine Panel B; Millipore, St. Charles, MN). Serum concentrations of adiponectin were quantified by enzyme-linked immunosorbent assay (Millipore).

Statistical analysis was performed using PASW Statistics version 18.0 (SPSS, Chicago, IL). Distribution of normality was tested using the one-sample Kolmogorov-Smirnov test. For within-group differences, a paired sample Student's $t$-test was used for normally distributed variables. For variables not normally distributed, a related-samples Wilcoxon signed-rank test was used. For differences between groups, the independent Student's $t$-test was used for variables with a normal distribution and the Mann-Whitney test for not normally distributed variables. To assess the association between changes over time of various variables, the difference over time was calculated as variable at baseline minus variable at $16 \mathrm{wk}$. All differences over time were normally distributed. Then, Pearson correlation coefficients were calculated. The significance level of all tests was 0.05. To further explore the underlying structure of the correlation in changes in cardiovascular risk factors after the obesity intervention, we performed exploratory factor analysis. Factors were attributed to variables with correlation $>0.55$, and variables with correlation $>0.3$ are mentioned.

\section{ACKNOWLEDGMENTS}

The authors thank Bettine G. Haandrikman for performing the enzymelinked immunosorbent assay analysis of the adipokines and markers for low-grade inflammation.

\section{STATEMENT OF FINANCIAL SUPPORT}

The study was supported with an unrestricted grant by Hutchison Whampoa Limited, 22/F Hutchison House, 10 Harcourt Road, Hong Kong. The study sponsor had no role in the design and conduct of the study; the collection, management, analysis, and interpretation of the data; the preparation, review, or approval of the manuscript; and the decision to submit the manuscript for publication.

Disclosure: The authors have nothing to disclose.

\section{REFERENCES}

1. Schönbeck Y, Talma H, van Dommelen P, et al. Increase in prevalence of overweight in Dutch children and adolescents: a comparison of nationwide growth studies in 1980, 1997 and 2009. PLoS ONE 2011;6:e27608.

2. Ebbeling CB, Pawlak DB, Ludwig DS. Childhood obesity: public-health crisis, common sense cure. Lancet 2002;360:473-82.

3. Zimmet P, Alberti KG, Kaufman F, et al.; IDF Consensus Group. The metabolic syndrome in children and adolescents - an IDF consensus report. Pediatr Diabetes 2007;8:299-306.

4. Martos R, Valle M, Morales RM, Cañete R, Gascón F, Urbano MM. Changes in body mass index are associated with changes in inflammatory and endothelial dysfunction markers in obese prepubertal children after 9 months of body mass index SD score loss. Metabolism 2009;58:1153-60.

5. Roth CL, Reinehr T. Roles of gastrointestinal and adipose tissue peptides in childhood obesity and changes after weight loss due to lifestyle intervention. Arch Pediatr Adolesc Med 2010;164:131-8.
6. Fontana L, Eagon JC, Trujillo ME, Scherer PE, Klein S. Visceral fat adipokine secretion is associated with systemic inflammation in obese humans. Diabetes 2007;56:1010-3.

7. Reinehr T, Stoffel-Wagner B, Roth CL, Andler W. High-sensitive C-reactive protein, tumor necrosis factor $\alpha$, and cardiovascular risk factors before and after weight loss in obese children. Metabolism 2005;54:1155-61.

8. Roth CL, Kratz M, Ralston MM, Reinehr T. Changes in adipose-derived inflammatory cytokines and chemokines after successful lifestyle intervention in obese children. Metab Clin Exp 2011;60:445-52.

9. Oude Luttikhuis $\mathrm{H}$, Baur L, Jansen $\mathrm{H}$, et al. Interventions for treating obesity in children. Cochrane Database Syst Rev 2009;CD001872.

10. Reinehr T, Roth C, Menke T, Andler W. Adiponectin before and after weight loss in obese children. J Clin Endocrinol Metab 2004;89:3790-4.

11. Bocca G, Corpeleijn E, Stolk RP, Sauer PJ. Results of a multidisciplinary treatment program in 3-year-old to 5-year-old overweight or obese children: a randomized controlled clinical trial. Arch Pediatr Adolesc Med 2012;166:1109-15.

12. Reinehr T, de Sousa G, Andler W. Longitudinal analyses among overweight, insulin resistance, and cardiovascular risk factors in children. Obes Res 2005;13:1824-33.

13. Ford AL, Hunt LP, Cooper A, Shield JP. What reduction in BMI SDS is required in obese adolescents to improve body composition and cardiometabolic health? Arch Dis Child 2010;95:256-61.

14. van der Heijden GJ, Wang ZJ, Chu ZD, et al. A 12-week aerobic exercise program reduces hepatic fat accumulation and insulin resistance in obese, Hispanic adolescents. Obesity (Silver Spring) 2010;18:384-90.

15. Berenson GS, Srinivasan SR, Bao W, Newman WP 3rd, Tracy RE, Wattigney WA. Association between multiple cardiovascular risk factors and atherosclerosis in children and young adults. The Bogalusa Heart Study. N Engl J Med 1998;338:1650-6.

16. Barlow SE; Expert Committee. Expert committee recommendations regarding the prevention, assessment, and treatment of child and adolescent overweight and obesity: summary report. Pediatrics 2007;120:Suppl 4:S164-92.

17. Hardy OT, Wiecha J, Kim A, et al. Effects of a multicomponent wellness intervention on dyslipidemia among overweight adolescents. J Pediatr Endocrinol Metab 2012;25:79-82.

18. Taubes G. Insulin resistance. Prosperity's plague. Science 2009;325:256-60.

19. Gustafson B, Hammarstedt A, Andersson CX, Smith U. Inflamed adipose tissue: a culprit underlying the metabolic syndrome and atherosclerosis. Arterioscler Thromb Vasc Biol 2007;27:2276-83.

20. Arner P. Insulin resistance in type 2 diabetes - role of the adipokines. Curr Mol Med 2005;5:333-9.

21. Weiss R, Kaufman FR. Metabolic complications of childhood obesity: identifying and mitigating the risk. Diabetes Care 2008;31:Suppl 2:S310-6.

22. Corpeleijn E, Saris WH, Blaak EE. Metabolic flexibility in the development of insulin resistance and type 2 diabetes: effects of lifestyle. Obes Rev 2009;10:178-93.

23. Pecoraro P, Guida B, Caroli M, et al. Body mass index and skinfold thickness versus bioimpedance analysis: fat mass prediction in children. Acta Diabetol 2003;40:Suppl 1:S278-81.

24. Stolk RP, Wink O, Zelissen PM, Meijer R, van Gils AP, Grobbee DE. Validity and reproducibility of ultrasonography for the measurement of intraabdominal adipose tissue. Int J Obes Relat Metab Disord 2001;25:1346-51.

25. Liem ET, De Lucia Rolfe E, L’Abée C, Sauer PJ, Ong KK, Stolk RP. Measuring abdominal adiposity in 6 to 7 -year-old children. Eur J Clin Nutr 2009;63:835-41.

26. Wallace TM, Levy JC, Matthews DR. Use and abuse of HOMA modeling. Diabetes Care 2004;27:1487-95. 